\title{
Inorganic phosphate is a trigger factor for Microbispora sp. ATCC-PTA-5024 growth and NAl-107 production
}

\author{
Anna Giardina ${ }^{1}$, Rosa Alduina ${ }^{1 *}$, Giuseppe Gallo ${ }^{1}$, Paolo Monciardini ${ }^{2,3}$, Margherita Sosio ${ }^{2,3}$ and Anna Maria Puglia ${ }^{1}$
}

\begin{abstract}
Background: NAl-107, produced by the actinomycete Microbispora sp. ATCC-PTA-5024, is a promising lantibiotic active against Gram-positive bacteria and currently in late preclinical-phase. Lantibiotics (lanthionine-containing antibiotics) are ribosomally synthesized and post-translationally modified peptides (RiPPs), encoded by structural genes as precursor peptides.

The biosynthesis of biologically active compounds is developmentally controlled and it depends upon a variety of environmental stimuli and conditions. Inorganic phosphate (Pi) usually negatively regulates biologically-active molecule production in Actinomycetes, while it has been reported to have a positive control on lantibiotic production in Firmicutes strains. So far, no information is available concerning the Pi effect on lantibiotic biosynthesis in Actinomycetes.
\end{abstract}

Results: After having developed a suitable defined medium, Pi-limiting conditions were established and confirmed by quantitative analysis of polyphosphate accumulation and of expression of selected Pho regulon genes, involved in the Pi-limitation stress response. Then, the effect of Pi on Microbispora growth and NAl-107 biosynthesis was investigated in a defined medium containing increasing Pi amounts. Altogether, our analyses revealed that phosphate is necessary for growth and positively influences both growth and NAI-107 production up to a concentration of $5 \mathrm{mM}$. Higher Pi concentrations were not found to further stimulate Microbispora growth and NAI-107 production.

Conclusion: These results, on one hand, enlarge the knowledge on Microbispora physiology, and, on the other one, could be helpful to develop a robust and economically feasible production process of NAl-107 as a drug for human use.

Keywords: Ribosomal Post-translationally modified Peptides (RiPPs), Phosphate, PhoP-PhoR, Polyphosphate

\section{Background}

Lantibiotics (lanthionine-containing antibiotics) are antimicrobial peptides produced by Gram-positive bacteria belonging to the Firmicutes and Actinobacteria phyla. These ribosomally synthesized and post-translationally modified peptides (RiPPs) are encoded by structural genes (generically named $\operatorname{lan} A$ ) as precursor peptides [1]. Before removal of an $\mathrm{N}$-terminal leader peptide and secretion, the precursor peptide undergoes modifications with the formation of meso-lanthionine (Lan) or 3-methyllanthionine

\footnotetext{
* Correspondence: valeria.alduina@unipa.it

'Department of Biological, Chemical and Pharmaceutical Sciences and Technologies, Università degli Studi di Palermo, Viale delle Scienze - Bd. 16, 90128 Palermo, Italy

Full list of author information is available at the end of the article
}

(Me-Lan) residues. This occurs via the dehydration of serine and threonine residues to dehydroalanine (Dha) and dehydrobutyrine $(\mathrm{Dhb})$ residues, respectively, then crosslinked via a thioether linkage with cysteine residues [1]. All the genes required for lanthipeptide biosynthesis are usually grouped in gene clusters, also containing genes whose products are involved in additional $\mathrm{C}$-terminal modification, pathway-specific regulation, lantibiotic export and cell immunity [2]. The biosynthesis of many lanthipeptides, e.g. nisin [3], actagardine [4] and NAI-107 [5-7], was genetically characterized. NAI-107, also known as microbisporicin or 107891, is a potent and promising lantibiotic produced by Microbispora sp. ATCC-PTA-5024 as a complex of related molecules, with the most abundant congeners, $A_{1}$ and $A_{2}$, differing by the presence of di-hydroxy- or 
hydroxy-proline at position 14 and containing a halogenated Trp residue [8]. It is active against Gram-positive bacteria, including methicillin-resistant Staphylococcus aureus (MRSA), glycopeptide-intermediate S. aureus (GISA) and vancomycin-resistant enterococci (VRE). It has shown superior efficacy in animal models of multidrug resistant infections compared with the drugs of last resort, linezolid and vancomycin [9]. It is currently in late preclinicalphase. The mlb and mib clusters, containing genes encoding the proteins required for NAI-107 biosynthesis in $M$. sp. ATCC-PTA-5024 and M. corallina NRRL 30420, respectively $[6,10]$, are essentially identical.

In M. corallina NRRL 30420 it was proposed that an unknown signal, possibly nutrient limitation, activates the positive regulator MibR in a growth rate-dependent manner. MibR triggers the expression of the mibABCD$T U V$ operon, leading to the precursor peptide biosynthesis $(m i b A)$, the core peptide modification (mibBCDV) and mature peptide proteolysis and export (mibTU). The precursor peptide export would cause release of $\sigma^{\text {MibX }}$ through the inactivation of the anti- $\sigma$ factor MibW. $\sigma^{\text {MibX }}$ controls, in addition to mibR, genes to confer immunity to microbisporicin ( $m i b E F$ and $m i b Q$ ) and genes required for tryptophan chlorination (mibHS) and proline hydroxylation (mibO), resulting in the formation of fully processed and active microbisporicin [7].

The biosynthesis of biologically active compounds is generally elicited as developmental program and physiological response to a variety of environmental stimuli and conditions, such as the nature and/or quantity of carbon, nitrogen and phosphate sources [11,12]. Inorganic phosphate $(\mathrm{Pi})$ usually regulates antibiotic biosynthesis negatively in both producing Actinomycetes [11,13,14] and engineered strains [15]. In Streptomycetes, cellular response to Pi-limitation stress is controlled by PhoR-PhoP Two Component System (TCS), in which PhoR is a membrane sensor kinase and PhoP is a DNA-binding response regulator. Under Pi limitation, phosphorylated PhoP is able to bind to PHO boxes upstream target genes and controls the expression of pho regulon genes. PhoP regulates genes coding for the alkaline phosphatase PhoD, phosphate transporter system PstSCAB and polyphosphate kinase (Ppk), which function in the scavenging of Pi, its transport and its storage as cellular polyphosphates [16,17]. Polyphosphates (PPs) are linear Pi polymers containing high-energy phosphoanhydride bonds. In vitro, they are synthesized when the ATP/ADP ratio is high and degraded when this ratio is low [18]. Both enzymatic activities, polyphosphate kinase (Ppk) and nucleoside diphosphate kinase (NDPK), can reside in the same protein, e.g., PPK2 in Pseudomonas aeruginosa [19]. In addition, Pi limitation positively regulates the antibiotic biosynthesis by exerting a control on regulatory genes of actinorhodin and undecilprodigiosin biosynthesis [20]. To the best of our knowledge, no information is available concerning the role of $\mathrm{Pi}$ on RiPP production in actinomycetes, although it has been reported that Pi positively influences lantibiotic production in Lactococcus lactis and Micrococcus sp. GO5 [21,22].

In this study, the effect of Pi on growth and NAI-107 production was investigated at biochemical and molecular genetic levels.

\section{Results and discussion}

\section{Design of a defined medium}

Starting from the mineral composition of MaltoseGlutamate (MG) medium [23], already used for model streptomycetes, such as Streptomyces coelicolor [24] and Streptomyces lividans [25,26], and other actinomycetes, such as Amycolatopsis balhimycina [27,28], four media were developed all containing Glucose (20 or $50 \mathrm{~g} / \mathrm{l}$ ) instead of maltose as carbon source and $60 \mathrm{mM}$ Glutamate (GG20 and GG50) or $25 \mathrm{mM}$ ammonium Nitrate (NG20 and NG50) as nitrogen source. Cell dry weight (CDW) and NAI-107 production were monitored every $24 \mathrm{~h}$ till $96 \mathrm{~h}$ (Additional file 1: Figure S1). NAI-107 production was verified by bioassay (Additional file 1: Figure S1B) and the presence of both NAI-107 congeners was confirmed by LC-MS analysis (data not shown). All media supported Microbispora growth, with medium NG-20 yielding both maximum biomass and maximum production of both congeners of NAI-107. Thus, this medium was used as the defined medium in the following experiments.

\section{Phosphate role on Microbispora growth and NAI-107 production}

Microbispora growth and NAI-107 production were monitored in NG20 medium by varying inorganic phosphate $(\mathrm{Pi})$ concentrations: $0,0.1,0.5,5,15$ and $30 \mathrm{mM}$ (labelled P0, P0.1, P0.5, P5, P15 and P30). Microbispora did not grow in $\mathrm{P} 0$, indicating that $\mathrm{Pi}$ is a prerequisite for growth (data not shown). Glucose, phosphate, nitrate and growth (percent packed mass volume, PMV\%) were monitored for the five cultures (Figure 1). Our results indicate that increasing initial Pi concentration improved the cell growth rate (calculated between 24 and $72 \mathrm{~h}$ of growth); indeed, cell growth rate was 0.048, 0.072, 0.086 $\mathrm{PMV} \% / \mathrm{h}$ in $\mathrm{P} 0.1, \mathrm{P} 0.5, \mathrm{P} 5$, respectively. Initial Pi concentration higher than $5 \mathrm{mM}$ did not significantly change the growth rate (0.089 PMV\%/h in P15 and P30). Consumption of glucose and nitrate was similar in the five cultures. At the end of the five cultivations, approximately $50-70 \mathrm{mM}$ of glucose and $5-17 \mathrm{mM}$ of nitrate were left, indicating that carbon and nitrogen sources were not limiting for growth (Figure 1). On the contrary, Pi was almost completely depleted (less than $0.1 \mathrm{mM}$ ) after $48 \mathrm{~h}$ in the P0.1 and after $144 \mathrm{~h}$ in the P0.5 (as indicated by asterisks), whereas it was never depleted in the P5, P15 and P30 cultivations. 

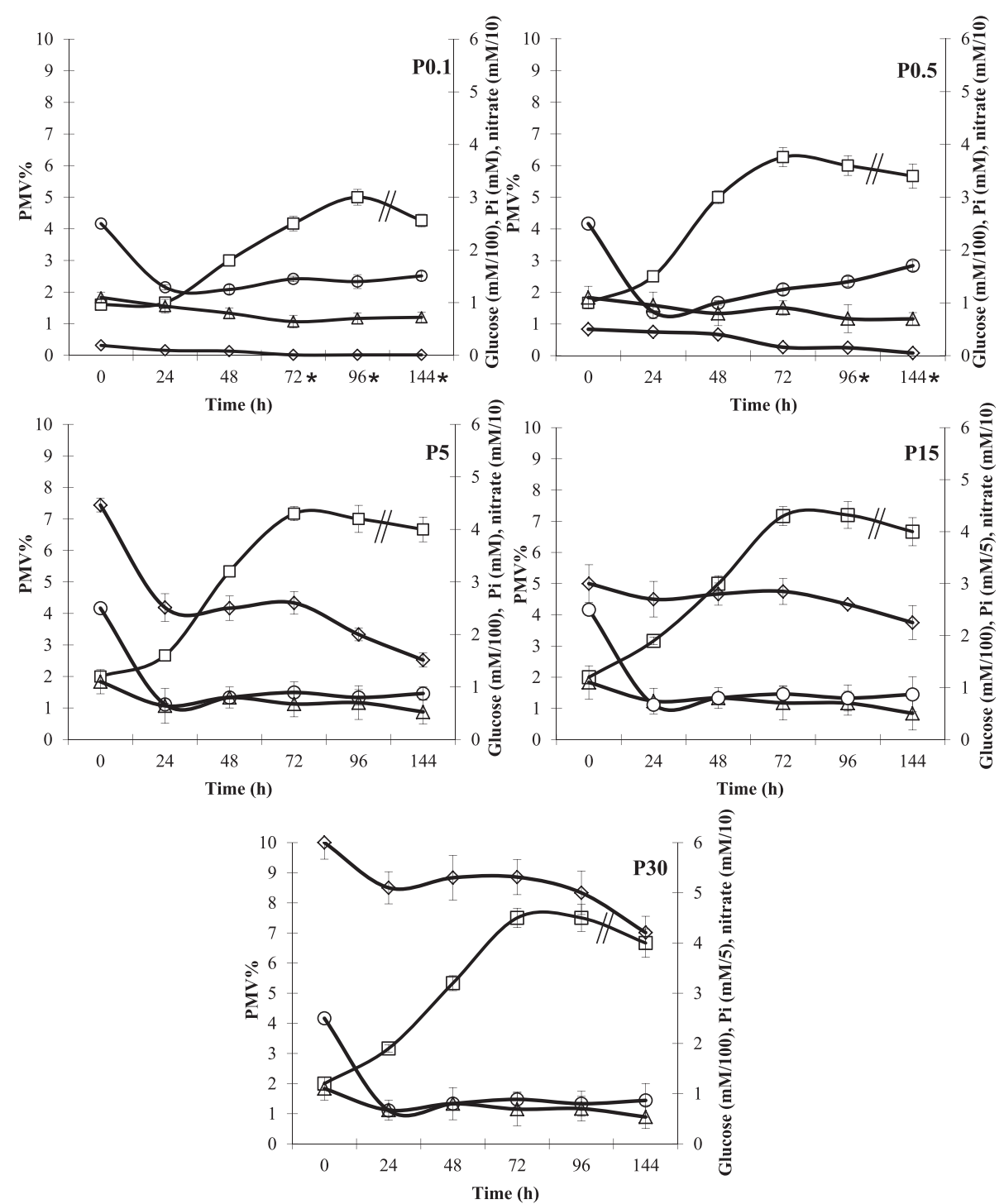

Figure 1 Microbispora sp. growth in P0.1, P0.5, P5, P15 and P30 media. PMV\% ( $\square)$, glucose $(\Delta)$, Pi $(\diamond)$ and nitrate (o) concentrations were monitored. Initial concentrations of glucose and nitrate were 110 and $25 \mathrm{mM}$, respectively. Initial Pi concentration ranged from 0.1 to $30 \mathrm{mM}$. Asterisks indicate the growth times where the phosphate concentration was lower than $0.1 \mathrm{mM}$. Standard deviation was calculated as average of three technical and two biological replicates. Cells were previously inoculated in GE82AB, incubated for $90 \mathrm{~h}$, washed three times, resuspended in distilled sterile water and used to inoculate the different media.

Microbiological assays revealed that NAI-107 was present after $48 \mathrm{~h}$ of growth in all cultures (Figure 2). Similarly to biomass accumulation profile, initial Pi concentration from 0.1 to $5 \mathrm{mM}$ stimulated NAI-107 production, and $\mathrm{Pi}$ concentrations higher than $5 \mathrm{mM}$ did not further stimulate production (asterisk in Figure 2). In actinomycetes, Pi is known as a key nutritional factor strongly influencing antibiotic production; i.e. biosynthesis of many non ribosomal peptide (NRP) antibiotics is repressed if the cultivation medium contains a $\mathrm{Pi}$ concentration higher than $4.2 \mathrm{mM}$ as for A40926 production in batch fermentation of Nonomuraea $[29,30,13]$ or $1.8 \mathrm{mM}$ as for balhimycin production in chemostat cultivation of
A. balhimycina [14]. By contrast, it has been reported that ribosomal biosynthesis of peptides such as nisin, micrococcin GO5, Pep5, epidermin, gallidermin is positively controlled by phosphate; indeed, nisin biosynthesis takes place at high initial phosphate concentrations up to $350 \mathrm{mM}$ and higher [21].

To determine that phosphate limitation had occurred, the accumulation of polyphosphates (PPs) and the expression of pho genes were analyzed in P0.1 and P0.5 (low phosphate containing media) and compared to those in P5 (one of the high phosphate containing media). PPs are mostly produced in a medium with adequate Pi concentration and are degraded to form ATP in a Pi-limited 


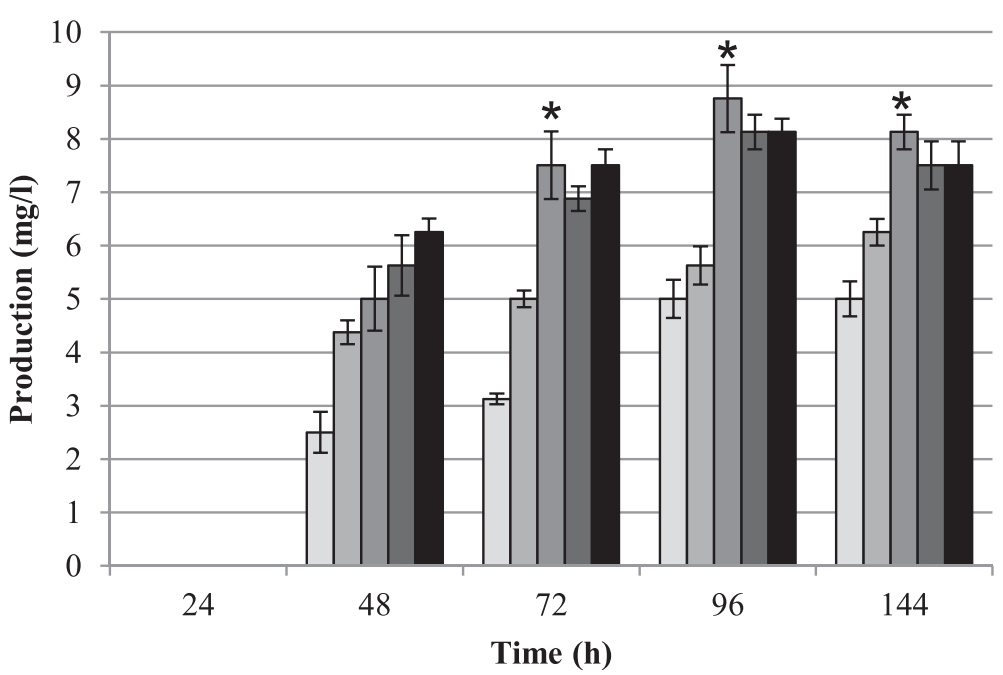

Figure 2 NAl-107 production in P0.1 (lighter gray bars), P0.5 (light gray bars), P5 (gray bars), P15 (dark gray bars) and P30 (black bars) media. The NAl-107 presence in the extracts was confirmed by LC-MS. The values were determined with a NAI-107 calibration curve (Additional file 1: Figure S3), as described in MM. Standard deviation was calculated from three independent bioassays. Asterisks indicate the phosphate concentration that allowed the maximum production.

medium [18] and can be used to monitor if the growth conditions are adequate or limiting of phosphate. As expected, in P0.1 and P0.5 Microbispora sp. did not accumulate PPs, while it accumulated PPs already after $16 \mathrm{~h}$ of growth in P5 (Figure 3A).

PhoR, PhoP, Ppk and PstS encoding genes were identified in the draft sequence of Microbispora sp. reported by Sosio et al. [10] by a Blast-P search using the homologues of Streptosporangium roseum and S. coelicolor A3(2). Microbispora PhoR (MPTA5024_26530), PhoP (MPTA5024_26525), Ppk (MPTA5024_37285) and PstS (MPTA5024_31955) show 84\% (59\%), 98\% (84\%), 84\% (50\%) and $80 \%(50 \%)$ sequence identity to the proteins of Streptosporangium roseum (or S. coelicolor). Sequence comparison indicated that Microbispora pho regulon genes have the same organization reported in S. coelicolor [17] and contain $\mathrm{PHO}$ box direct repeats (DRs) in their upstream region (Additional file 1: Figure S2A). The consensus of Microbispora DRs (Additional file 1: Figure S2B), created using free-on line available WebLogo software (http://weblogo.berkeley.edu/logo.cgi), shows high similarity with that of $S$. coelicolor [17]. The pho regulon genes, $p s t S$ and $p p k$, were previously used as reporters of $\mathrm{Pi}$ limitation in Streptomycetes [18,31-33].

Transcriptional analysis demonstrated that pstS and ppk gene expression was higher in the P0.1 and P0.5 cultures than in the P5 one (Figure 3B). phoR was weakly induced at $24 \mathrm{~h}$ (2.2- and 1.8-fold, respectively) and mainly induced at $72 \mathrm{~h}$ of growth (27- and 20-fold, respectively) in the P0.1 and P0.5 compared to the P5 culture and phoP transcription levels were almost similar in all the tested conditions. Altogether these results revealed that Pi was limiting in both P0.1 and P0.5.

Since in P0.5 medium, Microbispora showed a more similar growth rate to P5 than P0.1, P0.5 and P5 were chosen as the low and the high Pi condition, respectively.

\section{Effect of phosphate concentration on NAl-107 production and $m / b$ gene transcription}

To further confirm Pi effect on NAI-107 production, Microbispora was grown in P0.5 and P5, as representative conditions of low and high Pi. Biomass dry weight, nitrate, ammonium, $\mathrm{pH}$, glucose and phosphate were determined after $24 \mathrm{~h}$ and every $48 \mathrm{~h}$ over 9 days (Figure 4). As already demonstrated by Figure 1, the cell growth rate slightly increased in P5 (0.142 g/l*h) in respect to P0.5 (0.111 g/l*h) medium. The quantification of nitrate and ammonium showed that during the biomass accumulation phase (3 days of growth), both nitrogen sources were used (Figure 4C-D). Moreover, both carbon and nitrogen sources were not limiting for growth in both cultivations. At the end of the fermentation, phosphate was almost completely depleted in P0.5 cultivation, while a $2.8 \mathrm{mM}$ concentration was measured in P5 condition. Bioassays of spent medium from Microbispora cultures showed that NAI-107 production was detectable after three days of incubation in both P0.5 and P5 media, increased over the entire culture period and it was approximately 1.2-fold greater in the P5 than in the P0.5 medium (Figure 4A-B). It is noteworthy that productivity, calculated as a production/biomass ratio, was not influenced by Pi (Figure 4A-B), indicating that the difference in amount of lantibiotic produced in P0.5 

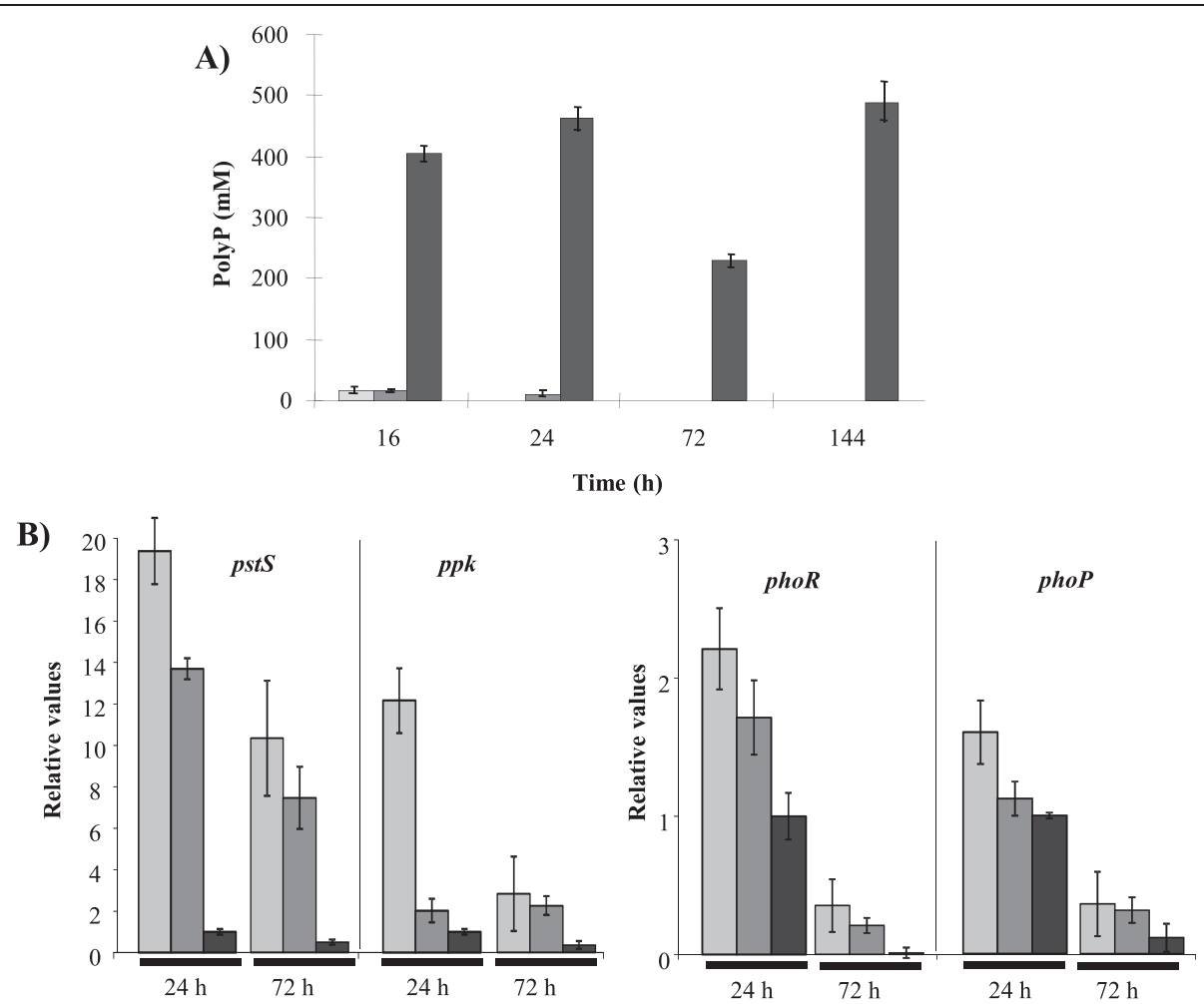

Figure 3 Pi content of extracted acid-soluble polyphosphates (ASPPs) from Microbispora sp. biomass (A) and transcriptional profile of selected Microbispora sp. pho genes (B) in P0.1 (light gray bars), P0.5 (dark gray bars) and P5 (black bars) media. Minimum and maximum relative values, calculated from three independent experiments, are reported as error bars. Relative values were normalized using $h r d B$ gene as endogenous gene.

and P5 medium was related to the difference in biomass yield. To evaluate Pi effect on transcription of $m l b$ genes, RNAs were extracted after 24 and $72 \mathrm{~h}$ of growth in P0.5 and P5 media and analyzed by Quantitative RT-PCR (Figure 5). The transcription levels of two regulatory genes, $m l b R$ and $m l b X$, the structural gene, $m l b A$, and genes coding for a modification enzyme, $m l b D$, and for an ABC-transporter, mlbE, were studied. As shown in Figure 5, Pi was found to activate transcription (3-fold) only of $m l b A$ and $m l b D$ at $24 \mathrm{~h}$ (indicated by asterisks), while it positively influenced the transcription of all the analyzed genes at $72 \mathrm{~h}$ (from 1.5-fold of $m l b D$ to 5.6-fold of $m l b R$ transcription). The high error bar of $m l b E$ transcript level could be due to a high heterogeneity in the expression of this gene at $24 \mathrm{~h}$.

Thus, in accordance with several reports concerning the positive effect of $\mathrm{Pi}$ on the biosynthesis of other ribosomal post-translationally peptides in Firmicutes (i.e. nisin, micrococcin GO5, Pep5, epidermin, gallidermin) [21-23], also NAI-107 production is positively Pi-controlled.

\section{Conclusion}

The present study provides experimental evidence for the positive effect of inorganic phosphate on Microbispora sp. ATCC-PTA-5024 growth and NAI-107 production.
NAI-107 is a Ribosomal Post-translationally modified Peptide (RiPP) currently in late preclinical-phase.

In the present study, different inorganic phosphate concentrations ranging from 0 to $30 \mathrm{mM}$ were used for Microbispora growth, demonstrating that phosphate is necessary and positively influences both growth (Figure 1) and NAI-107 production (Figure 2) up to a concentration of $5 \mathrm{mM}$. Higher Pi concentrations (15 and $30 \mathrm{mM}$ ) were not found to further stimulate Microbispora growth and NAI-107 production. In addition, $0.1 \mathrm{mM}$ and $0.5 \mathrm{mM}$ are limiting Pi concentrations, as demonstrated by analysis of PPs accumulation and of pho regulon gene transcription (Figure 3). Bioassay and LC-MS analysis demonstrated that $\mathrm{Pi}$ has a positive effect on NAI-107 production (Figures 2 and 4 ) and transcriptional analysis confirmed that selected $m l b$ genes, devoted to NAI-107 biosynthesis, were positively influenced by Pi mainly at $72 \mathrm{~h}$ (Figure 5). Our results are in accordance with several reports concerning the positive effect of $\mathrm{Pi}$ on the biosynthesis of other ribosomal post-translationally peptides in Firmicutes (i.e. nisin, micrococcin GO5, Pep5, epidermin, gallidermin) [21-23].

Detailed studies of the lantibiotic biosynthesis and investigation of the effects of other signals will help to understand the physiology of the producer strain and to 


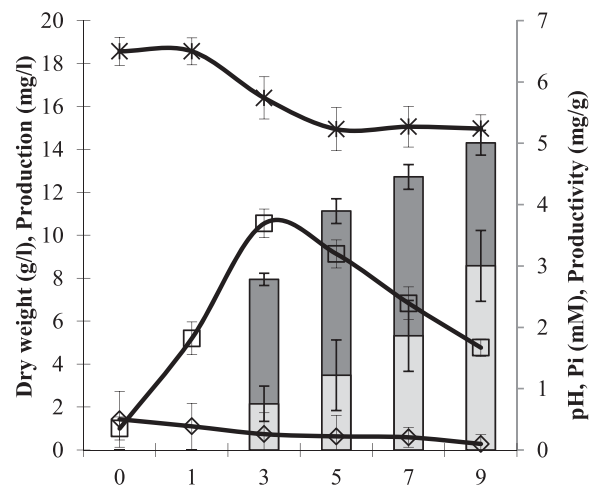

A)

Time (days)

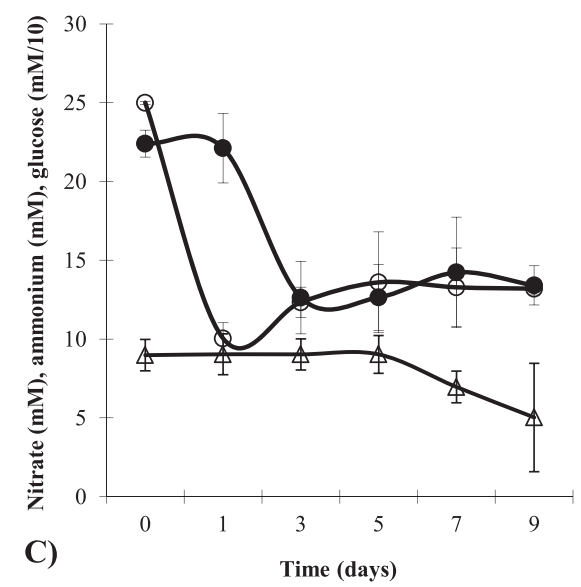

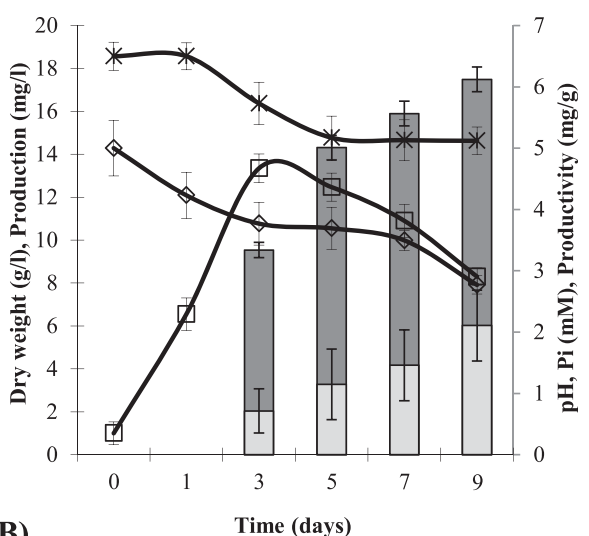

B)

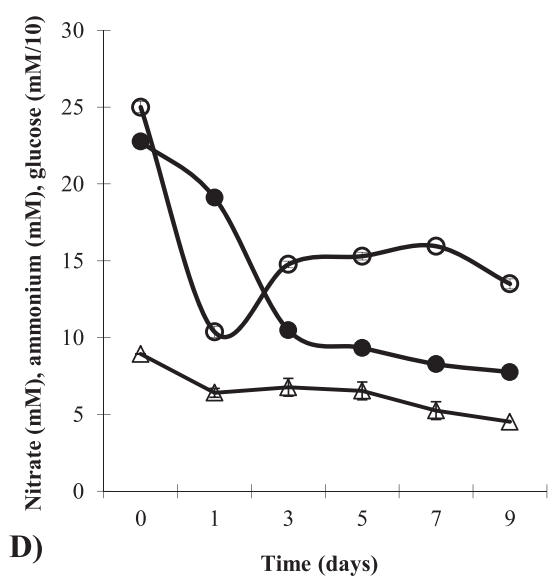

Figure 4 Microbispora sp. growth in P0.5 and P5 cultures. Cell dry weigth (white squares), NAI-107 production (dark gray bars), NAI-107 productivity (light gray bars), Pi (white diamonds) and pH (black crosses) of P0.5 (A) and P5 (B) cultures are shown. The values of production and productivity were standardized with a NAl-107 calibration curve (Additional file 1: Figure S3) as described in MM. Nitrates (white circles), ammonium (black circles) and glucose (white triangles) were monitored during the growth in P0.5 (C) and P5 (D) media. Initial concentrations of glucose, ammonium and nitrate were 110, 25 and 25 mM, respectively. Standard deviation was calculated as average of three technical and two biological replicates. The NAl-107 presence in the extracts was confirmed by LC-MS.

develop a robust and economically feasible production process.

\section{Methods}

\section{Strain and media}

\section{Microbispora sp}

ATCC-PTA-5024 was stored at $-80^{\circ} \mathrm{C}$ as frozen mycelium in GE82AB medium containing $20 \%$ glycerol at a biomass concentration of $6 \% \mathrm{w} / \mathrm{V}$ [8]. The composition of complex and chemically defined media used in this study is listed in Table 1.

\section{Growth conditions and media}

For seed culture preparations, $30 \mathrm{ml}$ of GE82AB medium in a $250-\mathrm{ml}$ baffled flask were inoculated with glycerol stock mycelium (6\%) and incubated with shaking (200 rpm) at $30^{\circ} \mathrm{C}$ for $72 \mathrm{~h}$. The resultant culture was subcultured in $30 \mathrm{ml}$ of GE82AB medium using a $6 \%$ inoculum. After
$90 \mathrm{~h}$ of incubation, the second seed culture (6\%) was used for inoculating $150 \mathrm{ml}$ of chemically defined medium in a 1 -L baffled flask. The culture was incubated at $30^{\circ} \mathrm{C}$ on a rotary shaker (200 rpm). When indicated (Figure 1), cells were washed three times with distilled sterile water and resuspended in $150 \mathrm{ml}$ of distilled sterile water prior to inoculate the defined media. Samples for determining growth and production of NAI-107 were withdrawn from the culture at different times. All cultivations were run in duplicate and all the measurements were done in triplicate. Standard deviations were calculated from the average of these values.

Biomass was determined by measuring the cell dry weight (CDW) of mycelial pellet recovered from a 1-ml culture sample after drying the pellet at $65^{\circ} \mathrm{C}$ for about $24 \mathrm{~h}$. Alternatively, it was determined by measuring the packed biomass volume in percent (PMV\%), obtained after centrifugation of a 6-ml culture sample for $10 \mathrm{~min}$ 


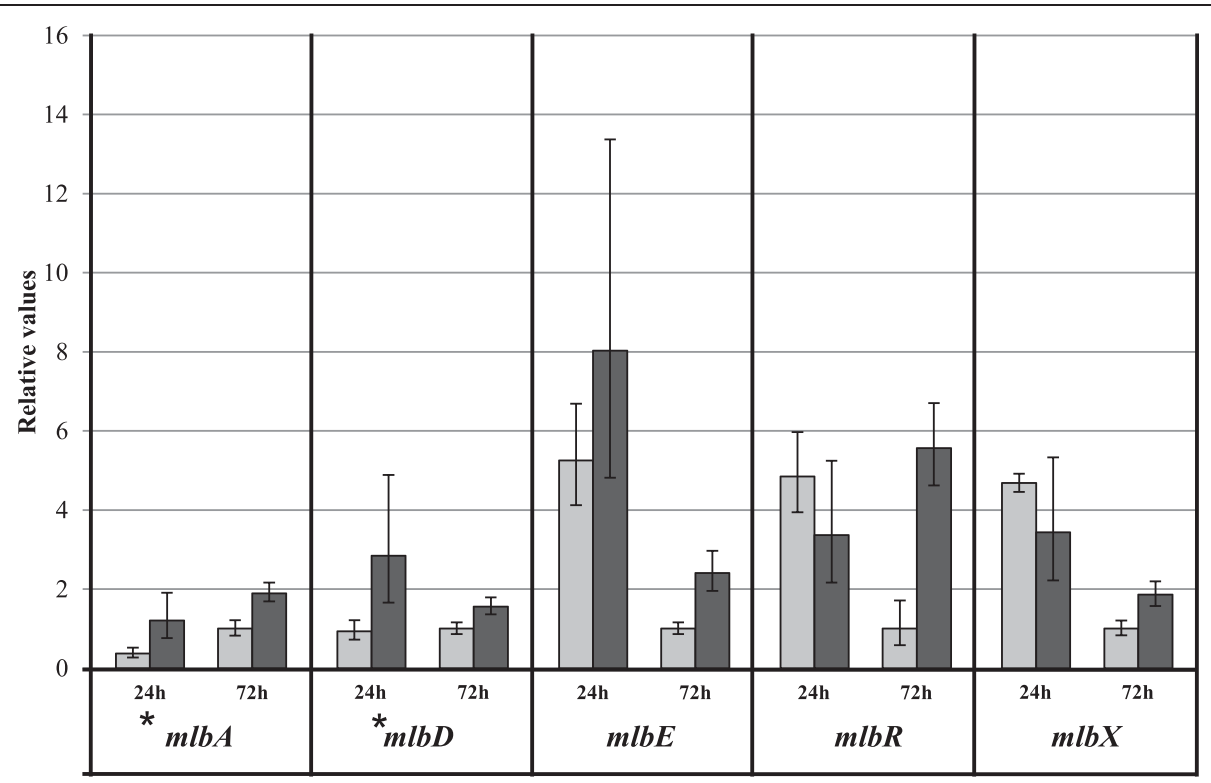

Figure 5 Quantitative RT-PCR of selected $\mathrm{m} / \mathrm{b}$ genes of Microbispora sp. after 24 and $72 \mathrm{~h}$ of incubation in P0.5 (light gray bars) and P5 (dark gray bars) media. Minimum and maximum relative values, calculated from three independent experiments, are reported as error bars. Relative values were normalized using hrdB gene as endogenous gene. Asterisks indicate genes slightly induced by Pi after $24 \mathrm{~h}$ of growth.

at 4000 rcf. Growth rates were calculated from the biomass increase between 24 and $72 \mathrm{~h}$ in the growing culture.

\section{Determination of Glucose, Nitrite, Nitrate, Ammonium, Phosphate and polyphosphate concentration}

Glucose, Nitrite and Phosphate concentrations were monitored during growth by electronic FreeStyle Freedom Lite $^{\bullet}$ Blood Glucose Monitoring System (Abbott), Griess Reagent Kit for Nitrite Determination (Invitrogen) and EnzCheck ${ }^{\oplus}$ Phosphate Assay Kit (Invitrogen), respectively.

Polyphosphates (PPs) were extracted by an acid method and determined as in [18]. Nitrate amount was measured as described in [34]. The determination of the ammonium ion concentration was performed by a spectrophotometric method using Nessler's reagent [35].

\section{Analysis of antibiotic production}

NAI-107 production was monitored by bioassay using the paper disc diffusion method with Micrococcus luteus ATCC 9341 [36] as test organism. For bioassay, $100 \mu \mathrm{L}$ of spent medium or extracts were used. The extracts were prepared by adding two volumes of methanol/ acetic acid (92/8 based on vol/vol) to $1 \mathrm{~mL}$ of culture and incubating at $50^{\circ} \mathrm{C}$ with shaking for $15 \mathrm{~min}$. The samples were then centrifuged (3000 rpm for $10 \mathrm{~min}$ ), and the supernatants were applied to Whatman $3 \mathrm{MM}$ Chr paper discs (Wathman, Maidstone, UK). After drying, wet discs were placed on the surface of LB soft agar inoculated with $100 \mu \mathrm{L} M$. luteus $(\mathrm{OD} 600=1.2)$. Inhibition zones were measured after over night (O.N.) incubation at $37^{\circ} \mathrm{C}$. A calibration curve using known concentrations of NAI-107 was constructed and used to calculate NAI-107 production

Table 1 Composition of media used for Microbispora fermentations

\begin{tabular}{|c|c|c|c|}
\hline Medium & Composition (g/L) & $\mathrm{pH}$ & References \\
\hline GE82AB & Maltodextrin (20), Soy flour (15), yeast extract (5), $\mathrm{CaCO}_{3}(1)$, Agar (1). & 7.3 & Monciardini, personal communication \\
\hline MG & $\begin{array}{l}\text { Maltose (20), Glutamate (11.23), MOPS (21), } \mathrm{MgSO}_{4}{ }^{*} 7 \mathrm{H}_{2} \mathrm{O}(0.2) \text {, } \\
\mathrm{FeSO}_{4}{ }^{*} 7 \mathrm{H}_{2} \mathrm{O}(0.09), \mathrm{CaCl}_{2}{ }^{*} 2 \mathrm{H}_{2} \mathrm{O}(0.001), \mathrm{NaCl}^{(0.001)} \text {, trace elements } \\
\text { (as described for R2YE medium), } 15 \mathrm{mM} \mathrm{PO}_{4} \text { buffer. }\end{array}$ & 6.5 & Puglia et al. [24] \\
\hline GG (20 or 50$)$ & $\begin{array}{l}\text { Glucose }(20 \text { or } 50), \text { Glutamate }(11.23), \mathrm{MOPS}(21), \mathrm{MgSO}_{4}{ }^{*} 7 \mathrm{H}_{2} \mathrm{O}(0.2) \text {, } \\
\mathrm{FeSO}_{4}{ }^{*} 7 \mathrm{H}_{2} \mathrm{O}(0.09), \mathrm{CaCl}_{2}{ }^{*} 2 \mathrm{H}_{2} \mathrm{O}(0.001), \mathrm{NaCl}(0.001), \text { trace elements } \\
\text { (as described for R2YE medium), } 15 \mathrm{mM} \mathrm{PO} \mathrm{PO}_{4} \text { buffer. }\end{array}$ & 6.5 & This study \\
\hline NG $(20$ or 50$)$ & $\begin{array}{l}\text { Glucose }(20 \text { or } 50), \mathrm{NH}_{4} \mathrm{NO}_{3} \text { (4), MOPS (21), } \mathrm{MgSO}_{4}{ }^{*} 7 \mathrm{H}_{2} \mathrm{O}(0.2) \text {, } \\
\text { FeSO }_{4}{ }^{*} 7 \mathrm{H}_{2} \mathrm{O}(0.09), \mathrm{CaCl}_{2}{ }^{*} 2 \mathrm{H}_{2} \mathrm{O}(0.001), \mathrm{NaCl}^{(0.001)} \text {, trace elements } \\
\text { (as described for R2YE medium), } 15 \mathrm{mM} \mathrm{PO}_{4} \text { buffer. }\end{array}$ & 6.5 & This study \\
\hline $\mathrm{P} 0, \mathrm{P} 0.1, \mathrm{P} 0.5, \mathrm{P} 5, \mathrm{P} 15$ and $\mathrm{P} 30$ & As NG20 with $0,0.1,0.5,5,15$ and $30 \mathrm{mM}$ of $\mathrm{PO}_{4}$ buffer, respectively. & 6.5 & This study \\
\hline
\end{tabular}


Table 2 Primers used in RT-PCR analysis

\begin{tabular}{|c|c|c|c|c|}
\hline Primer & Forward & Reverse & Amplicon size (bp) & Amplicon dissociation temperature $\left({ }^{\circ} \mathrm{C}\right)$ \\
\hline$h r d B$ & GATGCTCTTCCTGGACCTGA & CTTGTTGATCACCTCGACCA & 193 & 87.9 \\
\hline phoP & GACAGCGAGATCGACAAGGT & AGCAGCTCGAACTCCTTGAG & 239 & 91.6 \\
\hline phoR & ACCGTCGAGATAAGCGTCAG & CTTGCTCCATACCGTGACCT & 186 & 90.3 \\
\hline pstS & AGAACTCGCAGCTCCAGAAG & TCGTAGGTCACCAGGACGAT & 187 & 89.6 \\
\hline ppK & GACGTCACCGAGAACCTCAT & CAGGCGGTAGATCTCGTTGT & 159 & 88.9 \\
\hline$m / b A$ & GACTTCCGAGACCGAGGAC & GTGCACAGCGACCAAGCTC & 96 & 86.3 \\
\hline$m / b B$ & GCTGGTTCTTCATCCGCTAC & CTCGTACTCGGGGTCGTACT & 179 & 90.6 \\
\hline$m / b D$ & AACCCGGTGATGTGGCGCAAGC & CCGGCCGAGGCATGGTCAGTC & 148 & 90.7 \\
\hline$m / b E$ & CACGCTGGCGCTACTTCACCG & TCGAGGCGGCGTTGGAGAGCT & 129 & 90 \\
\hline$m / b Y$ & CGCCCTGCTCGTGTACGCCGT & GAACGCGGCGATCAGCAGTCC & 121 & 89.4 \\
\hline
\end{tabular}

(Additional file 1: Figure S3). Thus, productivity was deduced by normalizing the production for CDW. The presence of NAI-107 congeners in the extracts was verified by LC- MS as described in [8].

\section{Total RNA isolation and qRT-PCR analysis}

To perform transcriptional analysis of selected pho and $m l b$ genes, mycelium was harvested from $6 \mathrm{ml}$ of culture and resuspended in $1 \mathrm{ml}$ P-buffer containing lysozyme $(50 \mathrm{mg} / \mathrm{ml})$ and then incubated for $10 \mathrm{~min}$ at $37^{\circ} \mathrm{C}$. After two cycles of sonications (30 sec ON/20 sec OFF at output control 4 with Vibra cells, Sonics materials), RNA was extracted by using the RNeasy midi kit (Qiagen) according to the manufacturer's instructions. DNase I (Roche) treatment was performed at $37^{\circ} \mathrm{C}$ for $1 \mathrm{~h}$, and RNA was precipitated with 2 volumes of ethanol in the presence of 0.1 volume of $3 \mathrm{M}$ sodium acetate. After a washing step with $70 \%(\mathrm{v} / \mathrm{v})$ ethanol and air drying, the RNA pellet was resuspended in water with RNaseOUT $^{\mathrm{TM}}$ Recombinant Ribonuclease Inhibitor (Invitrogen). As control of RNA quality, a RT-PCR with $0.1 \mu \mathrm{g}$ of total RNA and primer pairs internal to $h r d B$, encoding a vegetative sigma factor, was carried out using the Superscript One-Step RT-PCR kit (Invitrogen) and the conditions indicated by the supplier. PCRs were performed on $0.5 \mu \mathrm{g}$ of RNA samples using 40 cycles to exclude the presence of genomic DNA. The identity of RT-PCR products was confirmed by sequencing. For qRT-PCR, a two step protocol was used. The high-capacity cDNA archive kit (Applied Biosystems) was used to retro transcribe $2 \mu \mathrm{g}$ of total extracted RNA. Primer pairs amplifying intragenic regions of the genes analyzed by qRT-PCR were designed with Primer3web version 4.0.0 (http://bioinfo.ut.ee/primer3/) to fulfill the following criteria: length of $20 \pm 2 \mathrm{bp}$, Tm of $60 \pm 1^{\circ} \mathrm{C}, \mathrm{GC}$ content superior to $50 \%$ and amplicon lengths ranging from 75 to $150 \mathrm{bp}$ (Table 2). Primer specificity was controlled by Blast analysis. Gene expression was analyzed quantitatively by using Applied Biosystems 7300 real-time
PCR system (Applied Biosystems) with $\mathrm{SYBR}^{\circ}$ Green PCR Master Mix (Applied Biosystems) in 96-well plates. Five $\mu \mathrm{L}$ of cDNA were added to $20 \mu \mathrm{L}$ of PCR. Amplification program consisted in an AmpErase ${ }^{\circ}$ UNG activation at $50^{\circ} \mathrm{C}$ for $2 \mathrm{~min}$, in a denaturation at $95^{\circ} \mathrm{C}$ for $10 \mathrm{~min}$ followed by 40 cycles at $95^{\circ} \mathrm{C}$ for $15 \mathrm{~s}$ and $60^{\circ} \mathrm{C}$ for $1 \mathrm{~min}$. Melting curves were performed from 60 to $95^{\circ} \mathrm{C}$ to validate the specificity of PCR reaction (Table 2). Three independent measurements were performed for each gene and culture condition. The threshold cycle values (CT) were determined with a baseline set automatically. Results were analysed using the comparative critical threshold method $(\triangle \triangle \mathrm{CT})$ in which the amount of target RNA is adjusted to an internal reference. $h r d B$ gene, encoding a vegetative sigma factor, did not show significant expression variation in these experiments (Additional file 1: Figure S4) and was used as an internal reference to normalize the results. Expression ratios were expressed as $2 \Delta \Delta \mathrm{CT}$. In each run, four dilutions of cDNA were analyzed to determine the PCR efficiency and negative controls were included. The amplicons obtained with each primer pair were sequenced.

\section{Additional file}

Additional file 1: Figure S1. Microbispora sp. growth (A) and NAl-107 production (B) in NG20 (black line), NG50 (black broken line), GG20 (light gray line) and GG50 (light gray broken line) media. + and - indicate the positive and negative control, respectively. Positive controls are supernatants of Microbispora grown in GE82AB and MG. An empty paper disk and a paper disk soaked in the corresponding defined medium were used as negative controls. Cells were previously inoculated in GE82AB, incubated for $90 \mathrm{~h}$ and directly used to inoculate the different media. Figure S2. Putative PHO box sequences found in upstream regions of Microbispora pho regulon genes (A). Translational start codons and transcription direction are indicated with bold letters and arrows, respectively. PHO boxes direct repeats (DRs) are underlined. B) Consensus of the direct repeats of $11 \mathrm{nt}$ that forms the Microbispora PHO box, obtained using free-on line available WebLogo software http://weblogo. berkeley.edu/logo.cgi. The height of each letter is proportional to the frequency of the base. Error bars are shown at the top of the stacks. Figure S3. Calibration curve constructed using known 
concentrations of NAl-107. Figure S4. Absolute Quantitative RT-PCR of hrdB using RNA extracted after 24 and $72 \mathrm{~h}$ of Microbispora sp. growth in P0.1 (light gray), P0.5 (dark gray) and P5 (black) media. Error bars were calculated from three independent experiments (A). The values were calculated using a standard curve obtained measuring fluorescence of known amounts $(10,100,1000,10000 \mu \mathrm{g})$ of c-DNA (B).

\section{Competing interests}

The authors declare that they have no competing interests.

\section{Authors' contributions}

AG analyzed Microbispora growth and NAl-107 production in the different Pi concentrations, verified Pi limiting conditions, carried out real-time RT-PCR of pho and mlb genes and wrote the draft manuscript. RA participated in the experimental design and coordination. GG performed bioinformatic analysis and participated in the experimental design. PM performed preliminary analysis for the choice of the minimal medium. MS carried out the chemical analysis. AMP supervised the study and participated in its coordination. RA, GG, PM, MS and AMP revised the manuscript. All authors read and approved the final manuscript.

\section{Acknowledgements}

This work was supported by the European Commission (contract no.245066 for FP7-KBBE-2009-3). The authors acknowledge all the colleagues participating in this project for stimulating discussion and helpful suggestions.

\section{Author details}

'Department of Biological, Chemical and Pharmaceutical Sciences and Technologies, Università degli Studi di Palermo, Viale delle Scienze - Bd. 16, 90128 Palermo, Italy. ${ }^{2}$ Naicons S.r.l., Viale Ortles 22/4, 20139 Milan, Italy.

${ }^{3}$ KtedoGen S.r.I., Viale Ortles 22/4, 20139 Milan, Italy.

Received: 26 May 2014 Accepted: 1 September 2014

Published online: 10 October 2014

\section{References}

1. Arnison PG, Bibb MJ, Bierbaum G, Bowers AA, Bugni TS, Bulaj G, Camarero JA, Campopiano DJ, Challis GL, Clardy J, Cotter PD, Craik DJ, Dawson M, Dittmann E, Donadio S, Dorrestein PC, Entian KD, Fischbach MA, Garavelli JS, Göransson U, Gruber CW, Haft DH, Hemscheidt TK, Hertweck C, Hill C, Horswill AR, Jaspars M, Kelly WL, Klinman JP, Kuipers OP, et al: Ribosomally synthesized and post-translationally modified peptide natural products: overview and recommendations for a universal nomenclature. Nat Prod Rep 2013, 30:108-160.

2. Chatterjee $C$, Paul M, Xie L, van der Donk WA: Biosynthesis and mode of action of lantibiotics. Chem Rev 2005, 105:633-684.

3. Siegers K, Heinzmann S, Entian KD: Biosynthesis of lantibiotic nisin. Posttranslational modification of its prepeptide occurs at a multimeric membrane-associated lanthionine synthetase complex. J Biol Chem 1996, 271(21):12294-12301.

4. Boakes S, Cortés J, Appleyard AN, Rudd BA, Dawson MJ: Organization of the genes encoding the biosynthesis of actagardine and engineering of a variant generation system. Mol Microbiol 2009, 72:1126-1136.

5. Castiglione F, Lazzarini A, Carrano L, Corti E, Ciciliato I, Gastaldo L, Candiani P, Losi D, Marinelli F, Selva E, Parenti F: Determining the structure and mode of action of microbisporicin, a potent lantibiotic active against multiresistant pathogens. Chem Biol 2008, 15(1):22-31.

6. Foulston LC, Bibb MJ: Microbisporicin gene cluster reveals unusual features of lantibiotic biosynthesis in actinomycetes. Proc Natl Acad Sci U S A 2010, 107(30):13461-13466.

7. Foulston L, Bibb M: Feed-forward regulation of microbisporicin biosynthesis in Microbispora corallina. J Bacteriol 2011, 193(12):3064-3071.

8. Maffioli SI, lorio M, Sosio M, Monciardini P, Gaspari E, Donadio S: Characterization of the Congeners in the Lantibiotic NAl-107 Complex. J Nat Prod 2014, 77(1):79-84.

9. Jabés D, Brunati C, Candiani G, Riva S, Romanó G, Donadio S: Efficacy of the new lantibiotic NAI-107 in experimental infections induced by multidrug-resistant Gram-positive pathogens. Antimicrob Agents Chemother 2011, 55(4):671-1676.
10. Sosio M, Gallo G, Pozzi R, Serina S, Monciardini P, Bera A, Stegmann E, Weber T: Draft genome sequence of the Microbispora sp. producing the lantibiotic NAI-107. Genome Announc 2014, 2(1):e01198-13.

11. Martin JF: Phosphate control of the biosynthesis of antibiotics and other secondary metabolites is mediated by the phoR-phoP system: an unfinished story. J Bacterio/ 2004, 186(16):5197-5201.

12. van Wezel GP, McDowall KJ: The regulation of the secondary metabolism of Streptomyces: new links and experimental advances. Nat Prod Rep 2011, 28(7):1311-1333.

13. Alduina R, Lo Piccolo L, D'Alia D, Ferraro C, Gunnarsson N, Donadio S, Puglia AM: Phosphate-controlled regulator for the biosynthesis of the dalbavancin precursor A40926. J Bacteriol 2007, 189(22):8120-8129.

14. Gallo G, Alduina R, Renzone G, Thykaer J, Bianco L, Eliasson-Lantz A, Scaloni A, Puglia AM: Differential proteomic analysis highlights metabolic strategies associated with balhimycin production in Amycolatopsis balhimycina chemostat cultivations. Microb Cell Fact 2010, 9:95.

15. Alduina R, Gallo G: Artificial chromosomes to explore and to exploit biosynthetic capabilities of actinomycetes. J Biomed Biotechnol 2012 2012:462049.

16. Chouayekh $\mathrm{H}$, Virolle MJ: The polyphosphate kinase plays a negative role in the control of antibiotic production in Streptomyces lividans. Mol Microbiol 2002, 43:919-930

17. Sola-Landa A, Rodríguez-García A, Franco-Domínguez E, Martín JF: Binding of PhoP to promoters of phosphate-regulated genes in Streptomyces coelicolor: identification of PHO boxes. Mol Microbiol 2005, 56(5):1373-1385.

18. Ghorbel S, Smirnov A, Chouayekh H, Sperandio B, Esnault C, Kormanec J, Virolle MJ: Regulation of ppk expression and in vivo function of Ppk in Streptomyces lividans TK24. J Bacteriol 2006, 188(17):6269-6276.

19. Ishige $K$, Zhang H, Kornberg A: Polyphosphate kinase (PPK2), a potent, polyphosphate-driven generator of GTP. Proc Natl Acad Sci U S A 2002, 99:16684-16688.

20. Fernández-Martínez LT, Santos-Beneit F, Martín JF: Is PhoR-PhoP partner fidelity strict? PhoR is required for the activation of the pho regulon in Streptomyces coelicolor. Mol Genet Genomics 2012, 287(7):565-573.

21. De Vuyst L, Vandamme EJ: Influence of the phosphorus and nitrogen source on nisin production in Lactococcus lactis subsp. lactis batch fermentations using a complex medium. Appl Microbiol Biotechnol 1993, 40:17-22.

22. Kim MH, Kong YJ, Baek H, Hyun $\mathrm{HH}$ : Optimization of culture conditions and medium composition for the production of micrococcin $\mathrm{GO} 5$ by Micrococcus sp. GO5. J Biotech 2006, 121:54-61.

23. Doull JL, Vining LC: Culture conditions promoting dispersed growth and biphasic production of actinorhodin in shaken cultures of Streptomyces coelicolor A3(2). FEMS Microbiol Lett 1989, 53(3):265-268.

24. Puglia AM, Vohradsky J, Thompson CJ: Developmental control of the heat-shock stress regulon in Streptomyces coelicolor. Mol Microbiol 1995, 17:737-746.

25. Alduina R, Giardina A, Gallo G, Renzone G, Ferraro C, Contino A, Scaloni A, Donadio S, Puglia AM: Expression in Streptomyces lividans of Nonomuraea genes cloned in an artificial chromosome. Appl Microbiol Biotechnol 2005 68(5):656-662.

26. Giardina A, Alduina R, Gottardi E, Di Caro V, Süssmuth RD, Puglia AM: Two heterologously expressed Planobispora rosea proteins cooperatively induce Streptomyces lividans thiostrepton uptake and storage from the extracellular medium. Microb Cell Fact 2010, 9:44.

27. Gallo G, Renzone G, Alduina R, Stegmann E, Weber T, Lantz AE, Thykaer J, Sangiorgi F, Scaloni A, Puglia AM: Differential proteomic analysis reveals novel links between primary metabolism and antibiotic production in Amycolatopsis balhimycina. Proteomics 2010, 10(7):1336-1358.

28. Alduina R, Gallo G, Renzone G, Weber T, Scaloni A, Puglia AM: Novel Amycolatopsis balhimycina biochemical abilities unveiled by proteomics. FEMS Microbiol Lett 2014, 351(2):209-215.

29. Gunnarsson N, Bruheim P, Nielsen J: Production of the glycopeptide antibiotic A40926 by Nonomuraea sp. ATCC 39727: influence of medium composition in batch fermentation. J Ind Microbiol Biotechnol 2003, 30(3):150-156.

30. Technikova-Dobrova Z, Damiano F, Tredici SM, Vigliotta G, di Summa R, Palese L, Abbrescia A, Labonia N, Gnoni GV, Alifano P: Design of mineral medium for growth of Actinomadura sp. ATCC 39727, producer of the 
glycopeptide A40926: effects of calcium ions and nitrogen sources. Appl Microbiol Biotechnol 2004, 65(6):671-677.

31. Santos-Beneit F, Rodríguez-García A, Franco-Domínguez E, Martín JF: Phosphate-dependent regulation of the low- and high-affinity transport systems in the model actinomycete Streptomyces coelicolor. Microbiol 2008, 154(8):2356-2370.

32. Rodríguez-García A, Barreiro C, Santos-Beneit F, Sola-Landa A, Martín JF: Genome-wide transcriptomic and proteomic analysis of the primary response to phosphate limitation in Streptomyces coelicolor M145 and in a $\Delta$ phoP mutant. Proteomics 2007, 7(14):2410-2429.

33. Thomas L, Hodgson DA, Wentzel A, Nieselt K, Ellingsen TE, Moore J, Morrissey ER, Legaie R, Consortium STREAM, Wohlleben W, Rodríguez-García A, Martín JF, Burroughs NJ, Wellington EM, Smith MC: Metabolic switches and adaptations deduced from the proteomes of Streptomyces coelicolor wild type and phoP mutant grown in batch culture. Mol Cell Proteom 2012, 11(2):M111.013797.

34. Fisher M, Alderson J, van Keulen G, White J, Sawers RG: The obligate aerobe Streptomyces coelicolor A3(2) synthesizes three active respiratory nitrate reductases. Microbiol 2010, 156:3166-3179.

35. Krug FJ, Ruzicka J, Hansen EH: Determination of ammonium in low concentration with Nessler's reagent by flow-injection analysis. Analyst 1979, 104:47-54.

36. Kovacs G, Burghardt J, Pradella S, Schumann P, Stackebrandt E, Marialigeti K: Kocuria palustris sp. nov. and Kocuria rhizophila sp. nov., isolated from the rhizoplane of the narrow-leaved cattail (Typha angustifolia). Int I Syst Bacteriol 1999, 49:167-173.

doi:10.1186/s12934-014-0133-0

Cite this article as: Giardina et al:: Inorganic phosphate is a trigger factor for Microbispora sp. ATCC-PTA-5024 growth and NAl-107 production.

Microbial Cell Factories 2014 13:133.

\section{Submit your next manuscript to BioMed Central and take full advantage of:}

- Convenient online submission

- Thorough peer review

- No space constraints or color figure charges

- Immediate publication on acceptance

- Inclusion in PubMed, CAS, Scopus and Google Scholar

- Research which is freely available for redistribution 